\title{
Improved 3-D Particle Tracking Velocimetry with Colored Particles
}

\author{
Christian Bendicks ${ }^{1}$, Dominique Tarlet ${ }^{2,3}$, Christoph Roloff ${ }^{2}$, Robert Bordás ${ }^{2}$, Bernd Wunderlich ${ }^{2}$, \\ Bernd Michaelis ${ }^{1}$, Dominique Thévenin ${ }^{2}$
}

${ }^{1}$ Institute of Electronics, Signal Processing and Communication Engineering, University Magdeburg, Magdeburg, Germany;
${ }^{2}$ Institute of Fluid Dynamics and Thermodynamics, University Magdeburg, Magdeburg, Germany; ${ }^{3}$ Laboratoire de Thermocinétique,
Université de Nantes, Nantes, France.
Email: christian.bendicks@ovgu.de

Received January $13^{\text {th }}, 2011$; revised February $25^{\text {th }}, 2011$; accepted March $2^{\text {nd }}, 2011$.

\begin{abstract}
The present work introduces an extension to three-dimensional Particle Tracking Velocimetry (3-D PTV) in order to investigate small-scale flow patterns. Instead of using monochrome particles the novelty over the prior state of the art is the use of differently dyed tracer particles and the identification of particle color classes directly on Bayer raw images. Especially in the case of a three camera setup it will be shown that the number of ambiguities is dramatically decreased when searching for homologous points in different images. This refers particularly to the determination of spatial particle positions and possibly to the linking of positions into trajectories. The approach allows the handling of tracer particles in high numbers and is therefore perfectly suited for gas flow investigations. Although the idea is simple, difficultties may arise particularly in determining the color class of individual particle when its projection on a Bayer sensor is too small. Hence, it is not recommended to extract features from RGB images for color class recognition due to information loss during the Bayer demosaicing process. This article demonstrates how to classify the color of small sized tracers directly on Bayer raw images.
\end{abstract}

Keywords: Particle Tracking Velocimetry, Color Recognition, Artificial Neural Network, Photogrammetry

\section{Introduction}

Over the last 20 years Particle Tracking Velocimetry (PTV) has been established as an interesting tool for 3-D flow measurements, cf. [1,2] and references within. PTV relies on the stereoscopic recognition and subsequent tracking in time of small particles in an illuminated volume using a multi-camera system. In this way, the flow information is contained in reconstructed particle trajectories. Typical applications cover pure liquid flows, pure gas flows and multiphase configurations. Concerning gas-eous flows PTV measurements are still considered a major challenge. First, as a consequence of the high temporal and spatial resolution required by measurements in gas flows [3,4], it is necessary to employ a sufficiently high particle number density. Additionally, the trajectories have to be long enough for a Lagrangian flow analysis: interesting physical results can only be determined if long correlation lengths have been recorded [5-7].

A flow chart for a typical procedure of the measure- ment process is shown in Figure 1. Assuming the cameras are properly calibrated and corrected for lens distortion, the algorithm consists of five major modules that process the tasks:

1) Image pre-processing: Correction of inhomogeneous illumination and noise reduction.

2) Segmentation: Making a decision for each pixel whether it belongs to a particle or to the background.

3) Locating particle centers: Computing particle centers from results of segmentation.

4) Determination of 3-D coordinates and intrinsic properties: Determining spatial particle locations for each frame from corresponding locations in camera images and calibration data; detecting also properties like color in the present case.

5) Use of the obtained information in a post-treatment specific to Fluid Dynamics, for instance for Particle Tracking.

Ambiguities constitute the major problem. They occur first when trying to reconstruct the 3-D coordinates of particles out of different camera perspectives; ambiguities 


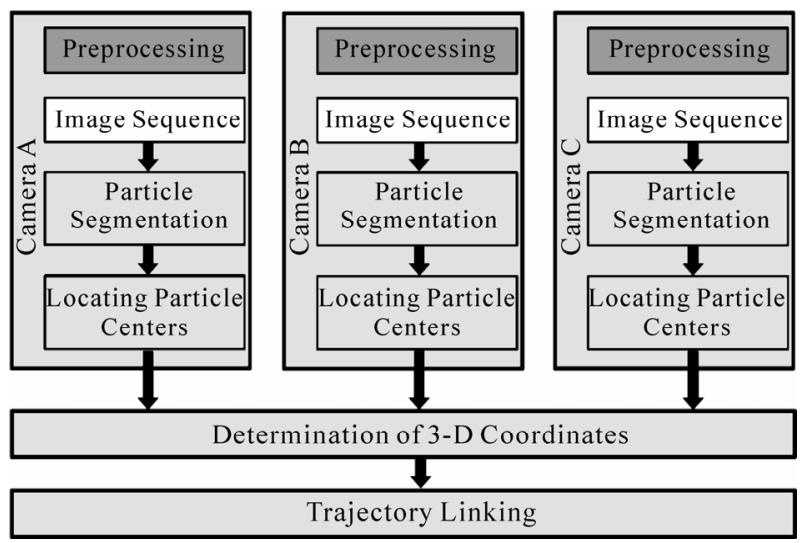

Figure 1. Usual design of a measurement system to perform particle tracking velocimetry.

are found again when assigning successive particle positions in time using the tracking algorithm (cf. Maas et al. in [8]). To reduce the number of ambiguities the simplest approach would be to use a low seeding density, but this would also reduce the spatial resolution of the system and the basis for an accurate analysis. A more sophisticated way of reducing ambiguities is to build classes of particles with distinctive properties, such as size [9], shape [10] or color (Figure 2). The first two criteria are not useful because proper particles for seed- ing in gases have to be very small to follow the flow. Using color as a distinctive criterion has already been proposed and investigated in detail by the research group of Prof. Brodkey $[11,12]$. Colored particles of a diameter of 38-44 $\mu \mathrm{m}$ were recorded on a $16 \mathrm{~mm}$ film and the pictures were digitized with considerable effort in several steps. To identify the color of a particle, particle pixels were averaged to create an RGB intensity vector which was compared to signature RGB vectors corresponding to one of the applied colors. Because of associated problems the use of color information has been found to be very difficult, if not impossible, using this post-processing strategy [12].

Instead of digitizing analog film material, today, digital high-speed cameras based on one CMOS or CCD sensor are commonly used to image the flow of particles. But since such type of sensor responds to all frequencies in the visible spectrum, it is unable to produce color images. Therefore, a color filter array is placed in front of the sensor of single chip color cameras, which allows only the light of a specific range of wavelengths to pass through each photodetector. The most commonly used color filter array is the Bayer filter array which is based on the human visual system. It consists of three kinds of filters, one for each primary color (red, green and blue). Green is sampled as twice the frequency of the other colors since the human visual system is most sensitive to the

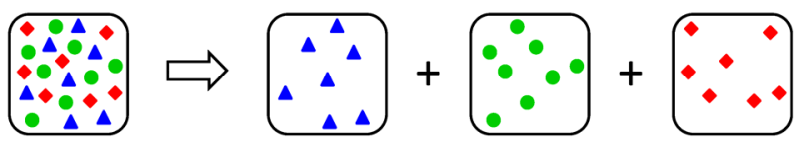

Figure 2. Reducing the apparent particle number density with the help of color classes.

green range of the spectrum. In addition, to ensure full image resolution, missing samples must be reconstructed through a process called demosaicing. This leads to significant color artifacts especially in high spatial frequency and/or small image structures. As a consequence, the assignment of a particle to its color class simply based on reconstructed RGB images is not reliable, when a particle projection covers only a few pixels.

In the following, the practical application of colored particles for digital PTV is demonstrated using classifycation of color classes prior to 3-D localization by the method of epipolar lines based on a three-camera setup. Note, in general the idea shown in Figure $\mathbf{2}$ is applicable for PTV experiments with two or more than three cameras. First, the experimental setup is described, including the choice of appropriate tracers and their coloring. An important step consists of image pre-processing and localization of particle centers on camera images. This information will subsequently be used for color classification, and will feed the correspondence solver to obtain 3-D localizations. The classification of small-sized colored particles $(\varnothing \approx 20 \mu \mathrm{m})$ is obtained by means of Artificial Neural Networks (ANNs). For the investigation of larger particles $(\varnothing \approx 70 \mu \mathrm{m})$, successful application of ANNs has been reported in [1]. The use of other classifyers like Support Vector Machines (SVM) is also possible and has been investigated by Bendicks et al. [13]. Here, ANNs have been retained because of the considerable experience of our group with this technique and of the huge amount of collected data for training and tests [14]. ANNs are highly flexible and are robust against slight variations in feature space. After the description of particle assignment to color classes, camera calibration is presented for the experimental setup. Then, the advantage of using color classes is shown in combination with the epipolar constraint for particle assignment among camera views. This is used as a basis for computing 3-D particle locations in each time frame. Quantitative measurement results (for example, proportion of successful correspondences with the same color) are finally discussed for three cases involving 3-D PTV.

\section{Experimental Setup: Particles and Imaging Method}

\subsection{Choosing and Dyeing Tracer Particles}

The material of the tracer particles is important since it 
determines their density $\rho_{p}$ and their agglomeration property. Ideally, a tracer particle of neutrally buoyant density is preferred. This is relatively easily realized in liquids $[5,6,15]$. In gas, smaller particle diameters are needed to keep the ability to follow the flow. However, the minimum diameter of the particles is limited by the production process and by optical considerations. A minimum number of pixels is needed on the camera sensor for each tracer particle to allow proper localization. In the present situation involving color recognition, the minimum number of pixels is about nine. Thus, a diameter of $20 \mu \mathrm{m}$ is at least required for the particles with the present setup. The physical properties of the tracer particles finally retained for the present study are reported in Table 1. The choice of suitable tracers is discussed in detail in other publications (see $[1,16]$ ).

Expanded Micro Spheres (EMS) have been finally identified as being the most suitable material due to appropriate size, low density, acceptable price, and good dyeing properties with low electrostatic loading. Dyeing of the particles is realized according to a standard, manual protocol. A small quantity $(70 \mathrm{mg})$ of EMS particles with a diameter of $20 \mu \mathrm{m}$ is introduced into a polyethylene container that has a volume of $4 \mathrm{~cm}^{3}$. Then, $250 \mathrm{mg}$ of a mix of $50 \%$ dye mass (in the present case Edding T25 liquid ink of color red, green or blue), and 50\% ethanol is added to the particles. This ethanol mix is realized in order to obtain a brighter color for the particles, in comparison with a non-diluted dye. Five minutes of careful mixing by means of a laboratory spatule ensures a homogeneous repartition of the dye through transport and diffusion within this porous medium. The dyed particles are then spread onto a cardboard layer for a day, during which they are turned three times. After drying the obtained colored particles are sieved through a metal filter of $25 \mu \mathrm{m}$ in size in order to remove possibly agglomerated particles. A microscopic check confirms that the particles have kept their original structure and that the increase in diameter is at most equal to $3 \mu \mathrm{m}$. This dyeing process results finally in a density of $320 \mathrm{~kg} / \mathrm{m}^{3}$ for the colored EMS particles with diameter of $23 \mu \mathrm{m}$. Only the three primary colors (red, green and blue) are used for the present study, allowing an easier recognition through a Bayer-Pattern sensor, composed of pixels of these 3 primary colors. The same method is nevertheless applicable to other colors as well as shown by further tests.

\subsection{Imaging of Particles in the Flow}

For suitable test measurements some small-sized (typical scale: $\mathrm{mm}$ ) flow patterns seeded with colored tracer particles have to be generated within the focal depth of the cameras. Figure 3 shows a drawing of the employed
Table 1. Properties of employed tracer particles.

\begin{tabular}{lcccc}
\hline \multirow{2}{*}{ Name } & \multicolumn{2}{c}{ Density $\left(\mathrm{kg} / \mathrm{m}^{3}\right)$} & \multicolumn{2}{c}{ Mean diameter $(\mu \mathrm{m})$} \\
\cline { 2 - 5 } & available & used here & available & used here \\
\hline Expancel ${ }^{*}(\mathrm{EMS})$ & $24-70$ & 70 & $20-120$ & 20 \\
Colored EMS & & 320 & & 23 \\
\hline
\end{tabular}

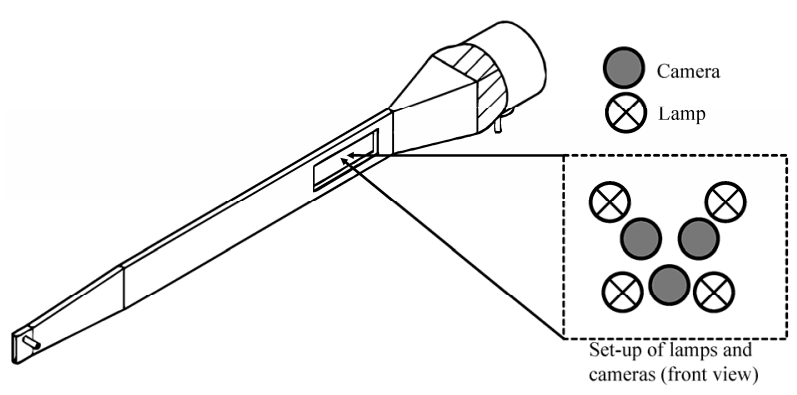

Figure 3. Perspective view of the eiffel wind tunnel used for all measurements.

wind tunnel and the arrangement of cameras and lighting in front of the observation window (an anti-reflective glass). The depth of the wind tunnel is $8 \mathrm{~mm}$ and is tailored to match the possible measurement depth ( $\mathrm{z}$ coordinate). The air flow is seeded with the tracer particles at its open extremity on the left (air intake). Beyond the right extremity (outflow), an electric fan entrains the air flow through a filter tissue with a pore size of $11 \mu \mathrm{m}$. This filter tissue captures the tracer particles and regularizes the flow induced by the fan in the wind tunnel. The optical setup is discussed in detail in the next section. Lamps and cameras are typically placed 20 to $30 \mathrm{~cm}$ away from the window giving optical access to the measurement section.

On top of the mainstream velocity, suitably tailored bluff bodies are placed in the measurement section to create a variety of flow properties. In Case 1 a set of three polyethylene winglets (length: $17 \mathrm{~mm}$, see Figure 4) simultaneously induce in the measurement volume large streamline curvature, continuous flow acceleration and small-scale recirculations. In Case 2, a transverse horizontal cylinder with a diameter of $6 \mathrm{~mm}$ creates a large recirculation zone downstream. These two flow patterns are mostly two-dimensional. To complete this experimental campaign of 3-D PTV, Case 3 relies on a rotating asymmetric device (width: $5 \mathrm{~mm}, 10$ rotations per second) leading to spiraling flow structures in the measurement section. In what follows, all aspects pertaining to color recognition and 3-D localization of the tracer particles are discussed.

To acquire the images three cameras (CMOS sensor with $1280 \times 1024$ pixels) are focused on the measurement section by means of a LINOS Apo-Rodagon D-2x 


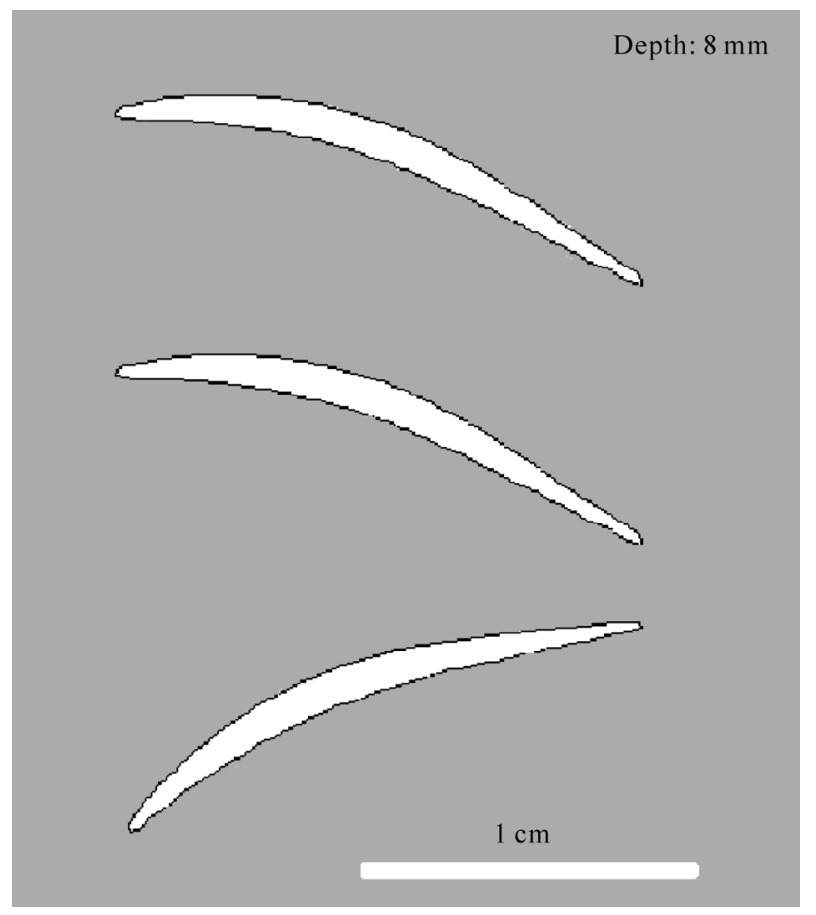

Figure 4. Set of three winglets (Case 1).

object lens with a focal length of $75 \mathrm{~mm}$ used at an f-number of 11 and located at a distance between 20 and $23 \mathrm{~cm}$ from the measurement section. For later calculations of 3-D coordinates, synchronization of the camera images - always acquired at $500 \mathrm{fps}$ during this project is of high importance. This synchronization is checked by simultaneously imaging a blinking light diode and verifying that illuminated states appear on the same frame. The cameras are designed to obtain a close-up image of the employed tracer particles with a diameter of $23 \mu \mathrm{m}$. Recording in monochrome mode enables to capture raw information from the CMOS sensor (Bayer Pattern of type G-R/B-G) that has a resolution of 1.3 megapixels $(1280 \times 1024)$. As later discussed, color recognition will be based on these Bayer raw data. Table 2 sums up the properties of the employed optical systems. It is important to note that the measurement section is of a size $25 \times 25 \times 8 \mathrm{~mm}$, which is less than the field of view of a 2-D image since all the areas of the 2-D images (especially the edges) have to intersect for 3-D localization.

For illumination four light heads are employed, based on halogen lamps emitting a light of color temperature between 3000 and $3400 \mathrm{~K}$. They are located at a distance of about $30 \mathrm{~cm}$ from the measurement section. Additionally, the light heads are equipped with hot mirrors and daylight filters, which are actively air-cooled to prevent loss of filtering properties resulting from excessive heat. In this way, the daylight filters and the measuring vol-
Table 2. Properties of the DEDOCOOL lamps and the BASLER A504kc cameras.

\begin{tabular}{|c|c|c|c|}
\hline \multicolumn{4}{|c|}{4 DEDOCOOL Lamps } \\
\hline \multicolumn{2}{|c|}{ Color temperature $(\mathrm{K})$ : } & \multicolumn{2}{|c|}{5300 (Halogen + Daylight filter) } \\
\hline Distance $(\mathrm{cm})$ : & 20 & 30 & 40 \\
\hline Light intensity (lux): & $24 \times 10^{6}$ & $1.1 \times 10^{6}$ & $0.58 \times 10^{6}$ \\
\hline Footcandle: & 222000 & 105000 & 54000 \\
\hline Illum. area $\varnothing(\mathrm{cm})$ : & 6.5 & 7 & 9 \\
\hline \multicolumn{4}{|c|}{3 synchronized BASLER 504kc cameras } \\
\hline Size of a pixel $(\mu \mathrm{m})$ : & $12 \times 12$ & Bayer Pattern: & G-R/B-G \\
\hline Sensor size $(\mathrm{mm})$ : & $15.36 \times 12.29$ & Resolution: & $1280 \times 1024$ \\
\hline f-Number: & 11 & Object dist. $(\mathrm{cm})$ : & $20-23$ \\
\hline Focal length $(\mathrm{mm})$ : & 75 & Depth of field (mm): & $\approx 6$ \\
\hline Recording rate (fps): & 500 & Exposure time (ms): & $0.8-1.0$ \\
\hline
\end{tabular}

ume are protected against heat produced by the illuminetion. Furthermore, the resulting illumination spectrum is similar to a light source with a color temperature of $5300 \mathrm{~K}$ (daylight). Therefore, Bayer sensor elements are not affected by near-infrared or reddish light, which would hinder color recognition. Properties of the lamps for different working distances are given in Table 2. Using three light heads placed between the cameras would lead to a more homogeneous illumination. However, tests revealed that the particle and color class recognition rate is higher with four light heads arranged as in Figure 3.

\section{Image Pre-Processing and Locating 2-D Particle Centers on an Image}

\subsection{Image Pre-Processing}

For each camera channel, an averaged image is first created over 20 consecutive images of the measurement section without seeding, which is later used to perform background subtraction on the entire Bayer image sequence. This ensures the removal of irrelevant structures like the winglets or dust deposition on the window. Since the algorithm employed to detect particle center considers grayscale images as input, Bayer images need to be converted in an intermediate step to RGB color images using a bilinear interpolation. This is performed with a demosaicing algorithm [17]. After that the images are converted to grayscaled ones using equal weights for red, green, and blue components to compute the intensity value.

The following pre-processing stage on grayscale images (introduced by Crocker and Grier [18]) deals with two problems complicating particle detection: 1) long 
wavelength modulations of the background intensity due to non-uniform sensitivity of the camera pixels or uneven illumination and 2) unavoidable digitization noise in the camera. To solve the first problem the background is removed by a boxcar average $(b a)$ over a square region with a side length of $(2 w+1)$ pixels:

$$
I_{b a}(x, y)=\frac{1}{(2 w+1)^{2}} \sum_{j=-w}^{w} \sum_{i=-w}^{w} I(x+i, y+j) .
$$

The user-defined parameter $w$ denotes a number of pixels larger than the apparent particle radius but smaller than the smallest interparticle separation. Digitization noise is modeled as uniformly Gaussian with a correlation length of $\lambda=1$ pixel. Thus, the convolution of image $I$ with a Gaussian surface results in the suppression of such noise without excessively blurring the image:

$$
I_{f}(x, y)=\frac{1}{b} \sum_{j=-w}^{w} \sum_{i=-w}^{w} I_{b a}(x+i, y+j) \exp \left(-\frac{i^{2}+j^{2}}{4 \lambda^{2}}\right)
$$

with normalization

$$
b=\left[\sum_{i=-w}^{w} \exp \left(-\frac{i^{2}}{4 \lambda^{2}}\right)\right]^{2} .
$$

The application of Equations (1) and (2) to image $I$ can be combined into one single convolution with the kernel

$$
K(i, j)=\frac{1}{K_{0}}\left[\frac{1}{b} \exp \left(-\frac{i^{2}+j^{2}}{4 \lambda^{2}}\right)-\frac{1}{(2 w+1)^{2}}\right] .
$$

The normalisation constant

$$
K_{0}=\frac{1}{b}\left[\sum_{i=-w}^{w} \exp \left(-\frac{i^{2}}{2 \lambda^{2}}\right)\right]^{2}-\frac{b}{(2 w+1)^{2}}
$$

can be used to compare filtered images with different values of $w$. The improved image is given by:

$$
I_{f}(x, y)=\sum_{j=-w}^{w} \sum_{i=-w}^{w} I(x-i, y-j) K(i, j) .
$$

\subsection{Locating 2-D Particle Centers}

To estimate particle center positions in a filtered image $I_{f}$ a connectivity analysis is performed as described by Maas [19]. This method can also handle overlapping particles. The goal is to find local maxima and assign surrounding pixels over a defined threshold to one maximum. Assigning a pixel to a maximum means assigning a pixel to an individual particle. The algorithm starts the search in the image pixel by pixel. When the pixel value is greater than a given threshold the pixel is tested to check if it is a local maximum. There is a local maximum when the pixel value is greater than or equal to the values of the eight neighboring pixels. If this is not the case, the search is continued. The local maximum is used as a seeding point for a region-growing operation in order to associate and label all pixels belonging to the particle. For this purpose, a so-called discontinuity threshold $D$ is introduced to decide if a pixel is assigned to a maximum or not. Suppose a pixel at location $p_{0}$ with intensity $I\left(p_{0}\right)$ is already assigned to a maximum and the exploration is continued at one of the unlabeled neighbors, called $p_{1}$ with intensity $I\left(p_{1}\right)$. Then, pixel $p_{1}$ is assigned to the current maximum if the following two conditions are true: 1) $I\left(p_{1}\right) \leq I\left(p_{0}\right)+D$ and (2) there are one or more $p_{n 1}$ which satisfy $I\left(p_{0}\right) \geq I\left(p_{n 1}\right)-D$, where $p_{n 1}$ identifies one of the other seven neighbors of $p_{1}$.

The operator described above considers the following rules:

1) All pixels belonging to a particle have values greater than the threshold.

2) A particle has exactly one local maximum.

3) The grayscale gradient within a particle projection is continuous.

4) A pixel, which represents a local minimum and could be assigned to several neighboring pixels, is assigned to the neighbor pixel with the largest value.

The second and the third rule can be weakened or strengthened by changing the discontinuity threshold $D$. Discontinuities up to $D$ grayscale values are tolerated within a single particle.

During the region-growing search process, the $n$ pixels belonging to the current local maximum are stored in a pixel list. Finally, particle coordinates are computed by the center of mass method:

$$
x=\frac{\sum_{i=0}^{n-1}\left(x_{i} \cdot I\left(x_{i}, y_{i}\right)\right)}{\sum_{i=0}^{n-1} I\left(x_{i}, y_{i}\right)}, y=\frac{\sum_{i=0}^{n-1}\left(y_{i} \cdot I\left(x_{i}, y_{i}\right)\right)}{\sum_{i=0}^{n-1} I\left(x_{i}, y_{i}\right)} .
$$

\section{Color Classification by an Artificial Neural Network (ANN)}

In the present application color classification is needed. Directly using RGB images created by a Bayer conversion algorithm and working with thresholds is not possible since this conversion leads to artifacts (see Figure 5). For our purpose, when attributing for instance the color "blue", the exact shade or brightness of the observed colored particle should not be taken into account since those will vary in time and space and differ slightly from particle to particle. As a consequence, the use of an Artificial Neural Network (ANN) is an appropriate solution for attributing particle color class [14]. ANNs are robust and able to learn and adapt according to training data associated with a given context. Other adequate classifyers like Support Vector Machines have been tested as well [13] but could not beat ANNs for the present appli- 
cation.

The proposed network design is a fully connected back-propagation network illustrated in Figure 6. It consists of

- an input layer with 10 neurons;

- a first hidden layer with 8 neurons;

- a second hidden layer with 8 neurons;

- an output layer with 3 neurons (corresponding to the number of applied color classes).

The number of output neurons is determined by the number of color classes used in the measurement experiment. Hence, the network has to be adapted when more or less colors are employed. All neurons in hidden layers and the output layer use sigmoid activation functions. In this way the network is able to make a predicttion based on fuzzy logic about the color class of a particle. The output neuron with the largest response value is considered as indicator of the particle color. In Figure 6 the outputs are denoted with $\mathrm{C}_{1}, \mathrm{C}_{2}$ and $\mathrm{C}_{3}$ corresponding respectively to blue, green and red color classes. This notation is introduced to remind the reader that these are only color classes that have been determined without considering the exact shade and brightness. In the present work all experiments are performed only with blue, green and red particles. Nevertheless, other tracer colors like cyan, magenta or yellow have been tested as well and are in principle suitable for further measurements.

The features used for network input are obtained directly from Bayer raw images ("input data", see Figure 6). Once a particle is localized on the grayscale image (as described in section 3.2) the values of the red, green and blue pixels (integer values of brightness between 0 and 255 - 8 bit) of the Bayer Pattern around its center are used to build the feature vector. The location of the center of the tracer particle given in integer coordinates $\left(x_{0}\right.$, $\left.y_{0}\right)$ is taken into account as being located either on a red, a blue or a green pixel of the Bayer Pattern. Green pixels are subdivided into two categories of green, due to their different localizations between red or blue pixels on the Bayer Pattern (cf. Figure 5). Finally, the feature vector includes ten components, the first one being a function $\Phi$ of the color associated with the center of the particle $\left(x_{0}\right.$, $\left.y_{0}\right)$ :

$$
\Phi\left(x_{0}, y_{0}\right)=\left\{\begin{array}{l}
0, \text { if }\left(x_{0}, y_{0}\right) \text { is Green } 1 \\
1, \text { if }\left(x_{0}, y_{0}\right) \text { is Red } \\
2, \text { if }\left(x_{0}, y_{0}\right) \text { is Blue } \\
3, \text { if }\left(x_{0}, y_{0}\right) \text { is Green } 2
\end{array}\right.
$$

The nine others are the intensity values $I(x, y)$ at the particle center pixel and at the eight neighboring pixels, in a clockwise scheme reported in Table 3 . As explained previously, the image of the tracer particle must cover a
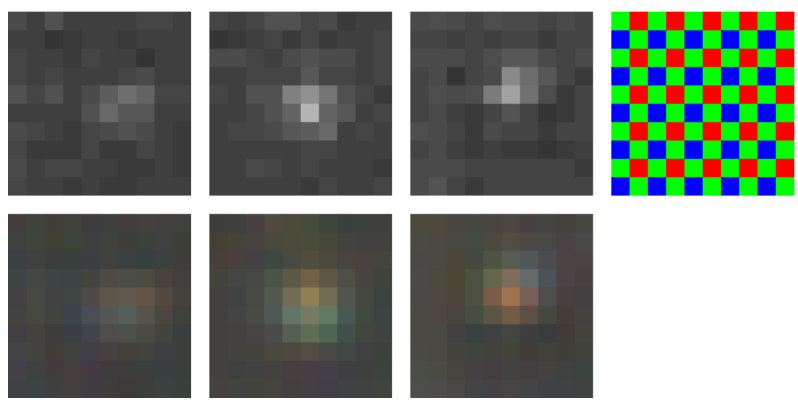

Figure 5. Appearance of different colored tracer particles $(23 \mu \mathrm{m})$ in Bayer images (upper row) and in resulting RGB images created by bilinear demosaicing (lower row). On the right there is the arrangement of color filters (Bayer pattern) on the CMOS-Chip. Color distortions appear on the RGB images as a consequence of demosaicing. Therefore, the RGB color space cannot be used for color classification.

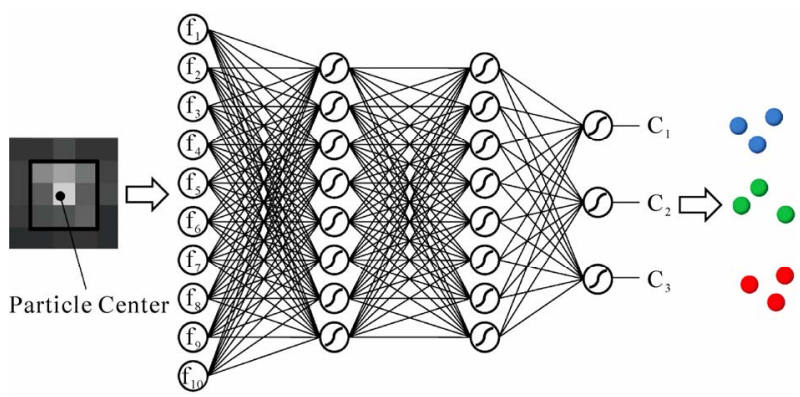

Figure 6. Principle of color classification with an Artificial Neural Network based on particle center position and surrounding Bayer data. Here, the network is designed for the use of three particle colors (therefore 3 output neurons).

Table 3. Feature vector definition.

\begin{tabular}{cc}
\hline Feature vector component & value \\
\hline $\mathrm{f}_{1}$ & $\Phi\left(x_{0}, y_{0}\right)$ \\
$\mathrm{f}_{2}$ & $I\left(x_{0}, y_{0}\right)$ \\
$\mathrm{f}_{3}$ & $I\left(x_{0}+1, y_{0}\right)$ \\
$\mathrm{f}_{4}$ & $I\left(x_{0}+1, y_{0}-1\right)$ \\
$\mathrm{f}_{5}$ & $I\left(x_{0}, y_{0}-1\right)$ \\
$\mathrm{f}_{6}$ & $I\left(x_{0}-1, y_{0}-1\right)$ \\
$\mathrm{f}_{7}$ & $I\left(x_{0}-1, y_{0}\right)$ \\
$\mathrm{f}_{8}$ & $I\left(x_{0}-1, y_{0}+1\right)$ \\
$\mathrm{f}_{9}$ & $I\left(x_{0}, y_{0}+1\right)$ \\
$\mathrm{f}_{10}$ & $I\left(x_{0}+1, y_{0}+1\right)$ \\
\hline
\end{tabular}

minimum area of $3 \times 3$ pixels in a Bayer Pattern in order to recognize particle color classes.

The training procedure consists in arranging the weights among the neural network (connections between neurons of adjacent layers, cf. Figure 6) in such a way that the 
color class resulting from the neural network is uniquely related to the actual color class of the particle. This color class is known in the case of training data, since three separate measurement passages are made in the wind tunnel, each with exclusively red, green, or blue particles. When acquiring training data, the measurement section has been fully equipped with the flow pattern device (i.e. winglets, cylinder or swirl generator). This ensures that the ANN is trained under the same flow and optical conditions as found during the later, actual measurements with a mix of colors.

After seeding the wind tunnel exclusively with red, green or blue EMS-particles of $23 \mu \mathrm{m}$ diameter, the image processing as well as localization of the tracer particles is realized as explained previously. At the end, the collected feature vectors constitute a training file composed (in each camera channel A, B and C) of 15000 feature vectors for each primary color class; this means 45000 feature vectors in a single training file for each camera channel. The training algorithm is based on the principle of Back Propagation [20] during 30000 training steps, so that a global squared error below $10 \%$ is finally attained.

The resulting ANN is then tested upon another set of 45000 feature vectors of each color, collected separately from the data used for training. For each color class, the percentage of correctly recognized vectors is called the recognition rate. Good results are obtained, with a typical recognition rate between 80 and $90 \%$, as detailed respecttively in Table 4 (Case 1: winglets), Table 5 (Case 2: cylinder), and Table 6 (Case 3: swirl generator). The recognition rate is only slightly lower than in experiments performed with larger particles $(\varnothing \approx 70 \mu \mathrm{m})$ as reported in [1] with a typical recognition rate of $85 \%$ $97 \%$.

Considering these results, a preference for some colors seems apparent among the mistakes. For example, green particles are mistaken with blue 5 or 10 times more often than with red. This point requires further investigations. The highest recognition rate is always for the red color. This could be attributed to the halogen lamps, emitting a color being slightly reddish in spite of the heat mirror. This would lead to a shift in the spectrum of light reflected by particle surface. Hence, the influence of blue and green Bayer pixel types in the feature vector would be reduced. Consequently the classification would mainly be based on intensity values of red pixel types, only. Also, in feature space the distance between green and blue color classes would be too small to separate these classes clearly by the neural network. To avoid this problem an ideal light source is needed, which emits all wavelengths of the visible spectrum in the same amount: white light. In respect of coloring particles it is important
Table 4. Performance of color recognition for each camera (Case 1: winglets).

\begin{tabular}{ccccc}
\hline Color class & Camera & $\mathrm{P}\left(\mathrm{C}_{1}\right)$ & $\mathrm{P}\left(\mathrm{C}_{2}\right)$ & $\mathrm{P}\left(\mathrm{C}_{3}\right)$ \\
\hline & $\mathrm{A}$ & $\mathbf{8 2 . 2 1} \%$ & $14.98 \%$ & $2.81 \%$ \\
$\mathrm{C}_{1}$ (blue) & $\mathrm{B}$ & $\mathbf{7 3 . 3 \%}$ & $23.94 \%$ & $2.76 \%$ \\
& $\mathrm{C}$ & $\mathbf{7 6 . 3 3 \%}$ & $22.07 \%$ & $1.6 \%$ \\
& $\mathrm{~A}$ & $23.48 \%$ & $\mathbf{7 2 . 1 \%}$ & $4.42 \%$ \\
$\mathrm{C}_{2}$ (green) & $\mathrm{B}$ & $10.66 \%$ & $\mathbf{8 4 . 0 \%}$ & $5.34 \%$ \\
& $\mathrm{C}$ & $11.5 \%$ & $\mathbf{8 0 . 8 2 \%}$ & $7.68 \%$ \\
& $\mathrm{~A}$ & $5.54 \%$ & $6.39 \%$ & $\mathbf{8 8 . 0 7 \%}$ \\
$\mathrm{C}_{3}$ (red) & $\mathrm{B}$ & $1.38 \%$ & $5.53 \%$ & $\mathbf{9 3 . 0 9 \%}$ \\
& $\mathrm{C}$ & $0.8 \%$ & $7.77 \%$ & $\mathbf{9 1 . 4 3 \%}$ \\
\hline
\end{tabular}

Table 5. Performance of color recognition for each camera (Case 2: cylinder).

\begin{tabular}{ccccc}
\hline Color class & Camera & $\mathrm{P}\left(\mathrm{C}_{1}\right)$ & $\mathrm{P}\left(\mathrm{C}_{2}\right)$ & $\mathrm{P}\left(\mathrm{C}_{3}\right)$ \\
\hline & $\mathrm{A}$ & $\mathbf{7 3 . 2 2 \%}$ & $24.84 \%$ & $1.94 \%$ \\
$\mathrm{C}_{1}$ (blue) & $\mathrm{B}$ & $\mathbf{6 8 . 6 9 \%}$ & $28.81 \%$ & $2.5 \%$ \\
& $\mathrm{C}$ & $\mathbf{8 3 . 0 5 \%}$ & $15.43 \%$ & $1.52 \%$ \\
$\mathrm{C}_{2}$ (green) & $\mathrm{A}$ & $15.1 \%$ & $\mathbf{8 2 . 7 6 \%}$ & $2.14 \%$ \\
& $\mathrm{~B}$ & $12.08 \%$ & $\mathbf{8 4 . 6 8 \%}$ & $3.24 \%$ \\
& $\mathrm{C}$ & $27.06 \%$ & $\mathbf{6 9 . 9 8 \%}$ & $2.96 \%$ \\
& $\mathrm{~A}$ & $2.44 \%$ & $4.93 \%$ & $\mathbf{9 2 . 6 3 \%}$ \\
$\mathrm{C}_{3}$ (red) & $\mathrm{B}$ & $1.89 \%$ & $6.42 \%$ & $\mathbf{9 1 . 6 9 \%}$ \\
& $\mathrm{C}$ & $6.59 \%$ & $7.48 \%$ & $\mathbf{8 5 . 9 3 \%}$ \\
\hline
\end{tabular}

Table 6. Performance of color recognition for each camera (Case 3: swirl generator).

\begin{tabular}{ccccc}
\hline Color class & Camera & $\mathrm{P}\left(\mathrm{C}_{1}\right)$ & $\mathrm{P}\left(\mathrm{C}_{2}\right)$ & $\mathrm{P}\left(\mathrm{C}_{3}\right)$ \\
\hline & $\mathrm{A}$ & $\mathbf{8 4 . 7 4 \%}$ & $14.59 \%$ & $0.67 \%$ \\
$\mathrm{C}_{1}$ (blue) & $\mathrm{B}$ & $\mathbf{9 0 . 1 8 \%}$ & $9.18 \%$ & $0.64 \%$ \\
& $\mathrm{C}$ & $\mathbf{7 9 . 9 3 \%}$ & $19.05 \%$ & $1.02 \%$ \\
$\mathrm{C}_{2}$ (green) & $\mathrm{A}$ & $5.97 \%$ & $\mathbf{9 3 . 0 5 \%}$ & $0.98 \%$ \\
& $\mathrm{~B}$ & $20.03 \%$ & $\mathbf{7 8 . 6 6} \%$ & $1.31 \%$ \\
& $\mathrm{C}$ & $7.92 \%$ & $\mathbf{9 0 . 4 7 \%}$ & $1.61 \%$ \\
$\mathrm{C}_{3}$ (red) & $\mathrm{A}$ & $0.54 \%$ & $1.58 \%$ & $\mathbf{9 7 . 8 8 \%}$ \\
& $\mathrm{B}$ & $1.39 \%$ & $2.32 \%$ & $\mathbf{9 6 . 2 9 \%}$ \\
& $\mathrm{C}$ & $0.83 \%$ & $2.54 \%$ & $\mathbf{9 6 . 6 3 \%}$ \\
\hline
\end{tabular}


to choose ink that can be distinguished well under the prevailing light conditions. This is all the more difficult the more color classes to be used.

\section{Locating Particle Centers in 3-D}

\subsection{Camera Calibration}

The purpose of calibration is the determination of all extrinsic (6 unknowns: location and orientation of the camera) and intrinsic (5 unknowns: principal point, calibrated focal length, lens distortion parameters) parameters of the used camera model. The mathematical formulation of the camera model is expressed by the collinearity equations which describe the transformation of 3-D world coordinates to 2-D image coordinates [21]. To compute all unknowns a set of well-known 3-D coordinates is needed (reference points), which can be mapped to their corresponding positions in camera images. For each reference point two equations are set up (one for each image coordinate). This leads to an overdetermined system of equations, solved by the least squares method.

For calibration a two-level calibration target (shown in Figure 7) with 25 reference points is used. In contrast to a plane target field, reference points are spatially distributed in all three dimensions and the calibration becomes more reliable [22]. First, the target is imaged in eight different orientations to determine the intrinsic parameters of each camera precisely. Then the target is placed in the center of the observation volume so that it faces the observation window. One snapshot is taken by each camera and is used to calibrate extrinsic parameters while keeping the intrinsic parameters fixed. This position of the calibration target determines the origin of the world coordinate system.

\subsection{Particle Assignment among Camera Views}

Figure 8 illustrates the whole process of solving the spatial correspondence problem for a single particle. The particle of interest is marked by a surrounding red square in Camera A - hence classified as a red one. Now, one

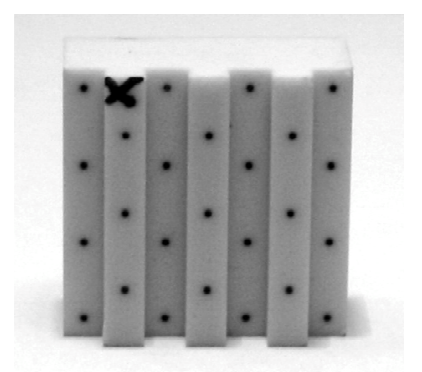

Figure 7. Two-level calibration target (dimensions: $20 \mathrm{~mm} \times$ $20 \mathrm{~mm} \times 5 \mathrm{~mm}$; level height: $2 \mathrm{~mm}$ ) with 25 reference points of known coordinates $(\varnothing=0.5 \mathrm{~mm})$.
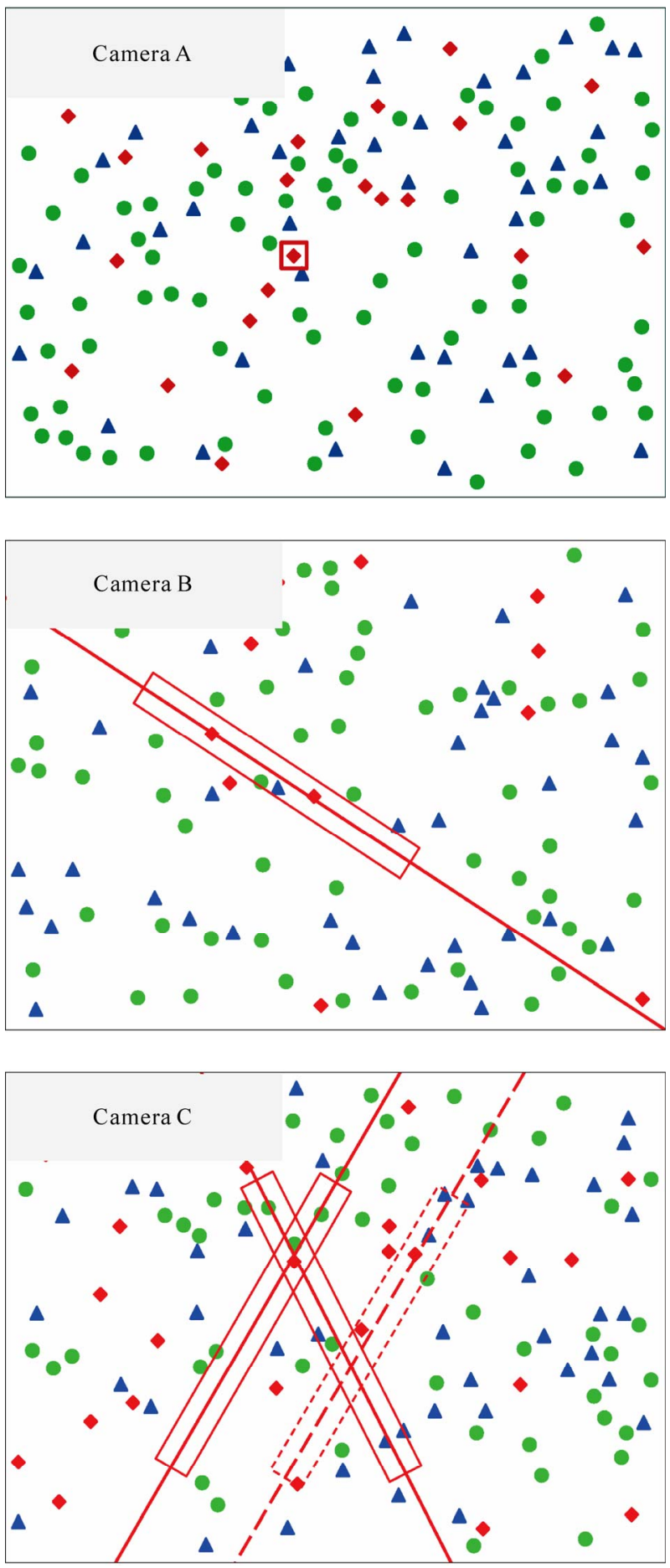

Figure 8. Reducing the number of ambiguities using epipolar geometry and color information.

corresponding partner should be found in Camera B and Camera $\mathrm{C}$. There are eight possible candidates near the epipolar line (red, from Camera A) which is constructed in Camera B, which means that their distance to the line is smaller than the tolerance value $\varepsilon$. Because the obser- 
vation volume is limited in depth ( $8 \mathrm{~mm})$, only a small segment of the epipolar line is considered. This leads to a further restriction of the search space, shown as a red box. Only four candidates are located in this red box and only two of them are classified to be red. In Camera $C$ an epipolar box is constructed from the chosen particle in Camera A, and two additional boxes are constructed from the red candidates in Camera B. When looking at the two intersection areas of the epipolar boxes, it is apparent that only one red particle can serve as a correspondence partner. If there is more than one candidate, the one with the smallest distance to one of the epipolar line intersections is chosen. The approach works very well as long as the tracer particles can be clearly assigned to their real color class.

The advantage of the proposed method is quantified in Table 7. The search for corresponding particles was performed as described above for four sample frames with different particle number densities obtained from the real, full-scale experiments. Here, $n$ denotes the number of particles in Camera $\mathrm{A}$ to which one or more possible correspondences have been finally found in Camera B and C. The columns $N_{a}$ and $N_{a c}$ contain the average number of ambiguities for each particle association when assuming all particles as monochrome (i.e., without considering color: $N_{a}$ ), respectively taking into account the color information $\left(N_{a c}\right)$. These real measurements differ a little bit from the theoretical calculations presented in the appendix (see later Figure 10) but the trend is very similar. Using the color information it is possible to reduce considerably the number of ambiguities, which is of the utmost importance for successful PTV measurements.

\subsection{Reliability of Color Class Recognition in Full-Scale Experiments}

The process employed to recognize a tracer particle is closely linked to the recognition of color classes. Indeed, the candidates should belong to the same color class when searching for correspondences in camera images $\mathrm{B}$ and $\mathrm{C}$ to a selected particle in $\mathrm{A}$. Nevertheless, in conflicting situations where only two of the three corresponding particles in the camera images are of the same color, the particle position in 3-D is assigned to this color class. If all three color classes are different, no 3-D position is assigned.

In Table 8, statistics resulting from three full-scale experiments associated with Case 1, 2 and 3 are presented. The number of possible matches in all three camera views, denoted here by $n$, is limited by the camera image containing the smallest number of detected particle centers for the selected frame. This number is further separated into the numbers of successful matches, where three $\left(n_{3 / 3}\right)$ or two $\left(n_{2 / 3}\right)$ of the three correspondences be-
Table 7. Measured number of ambiguities without $\left(N_{a}\right)$ and with $\left(N_{a c}\right)$ considering color classes.

\begin{tabular}{ccc}
\hline$n$ & $N_{a}$ & $N_{a c}$ \\
\hline 572 & 2.59 & 1.24 \\
756 & 3.34 & 1.28 \\
952 & 4.66 & 1.44 \\
1535 & 9.27 & 2.02 \\
\hline
\end{tabular}

Table 8. Matching particle color statistics in three camera views for the different configurations. The number of possible matches is denoted by $n$. The notation $n_{i / 3}$ is used for the number of matches where $\boldsymbol{i}$ of $\mathbf{3}$ correspondences belong to the same color class. The number of lost matches is denoted by $\boldsymbol{n}_{\text {lost }}$

\begin{tabular}{cccc}
\hline & Case 1: Winglets & Case 2: Cylinder & Case 3: Swirl \\
\hline$N$ & 1020 & 1478 & 929 \\
$n_{3 / 3}$ & 460 & 675 & 224 \\
$n_{2 / 3}$ & 455 & 694 & 554 \\
$n_{1 / 3}$ & 27 & 21 & 33 \\
$n_{\text {lost }}$ & 78 & 88 & 118 \\
\hline
\end{tabular}

long to the same color class. In this table the corresponddences associated with three different colors $\left(n_{1 / 3}\right)$ and therefore not considered further, and the number of lost matches, which cannot be determined at all by epipolar geometry $\left(n_{\text {lost }}\right)$ are also listed. In Cases 1 and 2, $n_{2 / 3}$ is similiar to $n_{3 / 3}$ due to the occurrence of overlapping particles on the different camera images. Case 3 (highly three-dimensional flow in the transverse direction) leads of course to a particularly challenging situation. Tracers in the foreground move transversally in the opposite direction compared with particles in the background. The probability of overlapping particle images rises and color classification becomes more difficult because of unknown/untrained particle patterns. One may notice in Table 8 that in all three Cases, epipolar associations with three different colors $\left(n_{1 / 3}\right)$ are very rare; about 2 to $4 \%$ only. This is one more indication of the reliability of the developed algorithm for color class recognition. Indeed, a failure of the algorithm is much more often the result of an impossible epipolar association $\left(n_{\text {lost }}\right)$ and not of a failed color association.

\subsection{Calculation of Spatial Tracer Coordinates}

The spatial location of a tracer $\boldsymbol{p}$ can be computed if homologous points are found in at least two different camera views. In this subsection the index $i=1, \cdots, 3$ is used to indicate the three different camera views. Note that because of small imprecisions due to the calculated coordi- 
nates of homologous points $\boldsymbol{s}_{i}$ and camera parameters the viewing rays do not necessarily intersect exactly in 3-D space. Accuracy and reliability increase when correspondences are found in all three camera views. The viewing rays from the homologous points $\boldsymbol{s}_{i}$ given in world coordinates on the sensor plane of camera $i$ in direction $\boldsymbol{d}_{i}=\boldsymbol{o}_{i}-\boldsymbol{s}_{i}$ to the projection center $\boldsymbol{o}_{i}$ should almost intersect at the spatial point $\boldsymbol{p}$ searched for, where $\boldsymbol{s}_{i}+t_{i} \boldsymbol{d}_{i}=\boldsymbol{p}$, i.e.:

$$
\left(\begin{array}{l}
x_{s i} \\
y_{s i} \\
z_{s i}
\end{array}\right)+t_{i}\left(\begin{array}{l}
x_{d i} \\
y_{d i} \\
z_{d i}
\end{array}\right)=\left(\begin{array}{c}
x \\
y \\
z
\end{array}\right) .
$$

Since there are three equations and one unknown, one still gets an overdetermined system of equations when performing a triangulation between two cameras only (6 equations $\rightarrow 3$ unknowns $x, y, z+2$ unknowns $t_{1}$ and $t_{2}$ ). If the solution is computed by adjustment of direct observations [23], this approach can easily be extended to three or more cameras. When rearranging (9) to

$$
\left(\begin{array}{c}
x \\
y \\
z
\end{array}\right)-t_{i}\left(\begin{array}{l}
x_{d i} \\
y_{d i} \\
z_{d i}
\end{array}\right)=\left(\begin{array}{l}
x_{s i} \\
y_{s i} \\
z_{s i}
\end{array}\right)
$$

the unknowns can be separated and (10) can be expressed in matrix form

$$
\left(\begin{array}{cccc}
1 & 0 & 0 & x_{d i} \\
0 & 1 & 0 & y_{d i} \\
0 & 0 & 1 & z_{d i}
\end{array}\right)\left(\begin{array}{c}
x \\
y \\
z \\
-t_{i}
\end{array}\right)=\left(\begin{array}{c}
x_{s i} \\
y_{s i} \\
z_{s i}
\end{array}\right) .
$$

Combining all viewing rays results in an overdetermined equation system of the form:

$$
A x=y .
$$

The design matrix is denoted by $\boldsymbol{A}$, vector $\boldsymbol{x}$ contains all unknowns $x, y, z$ and the negative scaling parameters $-t_{i}$, and vector $y$ contains all observations of $x_{s i}, y_{s i}$ and $z_{s i}$. If there are three corresponding points the system of equations results in

$$
\left(\begin{array}{cccccc}
1 & 0 & 0 & x_{d 1} & 0 & 0 \\
0 & 1 & 0 & y_{d 1} & 0 & 0 \\
0 & 0 & 1 & z_{d 1} & 0 & 0 \\
1 & 0 & 0 & 0 & x_{d 2} & 0 \\
0 & 1 & 0 & 0 & y_{d 2} & 0 \\
0 & 0 & 1 & 0 & z_{d 2} & 0 \\
1 & 0 & 0 & 0 & 0 & x_{d 3} \\
0 & 1 & 0 & 0 & 0 & y_{d 3} \\
0 & 0 & 1 & 0 & 0 & z_{d 3}
\end{array}\right)\left(\begin{array}{c}
x \\
y \\
z \\
-t_{1} \\
-t_{2} \\
-t_{3}
\end{array}\right)=\left(\begin{array}{c}
x_{s 1} \\
y_{s 1} \\
z_{s 1} \\
x_{s 2} \\
y_{s 2} \\
z_{s 2} \\
x_{s 3} \\
y_{s 3} \\
z_{s 3}
\end{array}\right) .
$$

Finally, the calculation of the unknowns in $\boldsymbol{x}$ can be performed using the least squares method:

$$
\boldsymbol{x}=\left(\boldsymbol{A}^{T} \boldsymbol{A}\right)^{-1}\left(\boldsymbol{A}^{T} \boldsymbol{y}\right) .
$$

\subsection{Experimental Determination of the Localization Uncertainty}

To get a reliable order of magnitude for the measuring error, a reference plate with circular 49 marks was fixed on a translation stage with manual adjustment of micrometer accuracy. It was placed inside the observation volume, so that all marks can be imaged from each camera. The 3-D coordinates of the marks were calculated as described above. The new 3-D positions of the marks were determined when the plate was moved $5.98 \mathrm{~mm}$ towards the cameras using the translation stage. Ideally, the shift should be the same for all 49 marks. In fact the measurement of 49 shifted marks resulted in a mean shift value of $5.98 \mathrm{~mm}$ and a standard deviation of $0.0069 \mathrm{~mm}$. The maximum measured shift within the set of marks was $5.9953 \mathrm{~mm}$ and the minimum was $5.9677 \mathrm{~mm}$. This results in a difference of $0.0276 \mathrm{~mm}$. This tiny value indicates that the uncertainty is below $0.5 \%$ in terms of distance between actual and measured localization.

To track the particles in time a 3 -frame algorithm based on Minimum Acceleration algorithm is employed $[2,24]$. The computation of trajectories is described in detail in a separate publication [25]. Figure 9 demonstrates the ability to obtain PTV data within the whole three-dimensional volume of observation around three winglets (Case 1). There is a factor 20 between the highest and the lowest measured velocity, which already represents a challenge for 3-D PTV [2].

\section{Conclusions and Perspectives}

Dense trajectory bundles have to be reconstructed to resolve small flow patterns in PTV experiments. Therefore, a high number of tracer particles is necessary. But this will increase the occurrence of ambiguities leading to problems when searching for corresponding particle locations in different camera views. The relative number of the particles can be reduced if particles can be distinguished e.g., by color. An original procedure has been developed to dye, localize in 3-D, and identify the color class of small tracer particles on Bayer raw images, used to investigate three different flow patterns by Particle Tracking Velocimetry. A post-processing strategy using Artificial Neural Networks combined with a standardized procedure for particle dyeing results in high color recognition rates, typically between 80 and $90 \%$. Furthermore, based on the huge amount of available training data Artificial Neural Networks are robust enough so that the developed procedure can be rapidly adapted to different conditions. The described method for classifying particle color is recommended when particle projections cover 


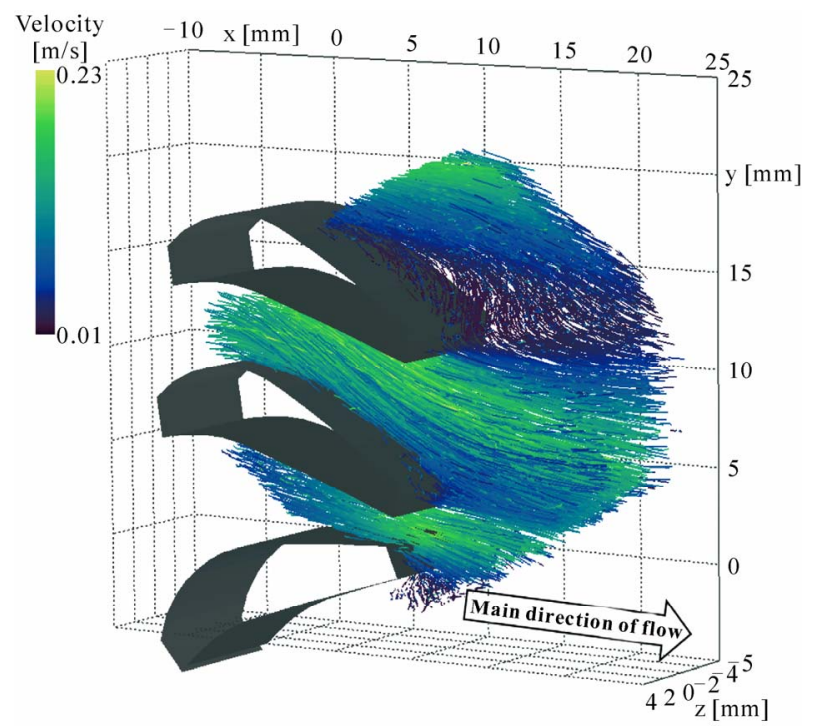

Figure 9. Visualization of trajectories in gas flow for case 1 (winglets).

only a few pixels. Then, classifying the color in RGB or HSI color space is erroneous, because of artifacts due to the demosaicing process. Finally, the feasibility of the particle recognition in 3-D gas flows has been demonstrated. Typically, 90\% of all tracer particles can be finally localized with high precision (spatial uncertainty about $0.5 \%$ ) using 3-D photogrammetry. When using color information, more tracers can be applied for seeding the flow, as required for PTV to resolve small-scale flow patterns.

In future measurements, further color classes (starting with yellow) will be added for seeding. Also, first tests involving smaller fluorescent tracer particles appear to be even more interesting, but also challenging. While the employed EMS particles are suitable for present conditions and slow gas flows with low Reynolds number, smaller particles are needed to investigate real turbulent flows with higher velocities. Furthermore, using fluorescence will eliminate the effect of specular reflection on the particle surface, which could be a reason for deteriorated color classification.

\section{Acknowledgements}

The authors would like to thank the DFG (Deutsche Forschungsgemeinschaft, Schwerpunktprogramm 1147) for the financial support of this project. Interesting discussions with R.V. Ayyagari, and T. Ruskowski are gratefully acknowledged.

\section{REFERENCES}

[1] C. Bendicks, D. Tarlet, B. Michaelis, D. Thévenin and B.
Wunderlich, "Coloured Tracer Particles Employed for 3-D Particle Tracking Velocimetry in Gas Flows," In: W. Nitsche and C. Dobriloff, Eds., Imaging Measurement Methods for Flow Analysis, Springer, Heidelberg, 2009, pp. 93-102.

[2] N. T. Ouelette, H. Xu and E. Bodenschatz, "A Quantitative Study of Three-Dimensional Lagrangian Particle Tracking Algorithms," Experiments in Fluids, Vol. 40, No. 2, 2006, pp. 301-313.

doi:10.1007/s00348-005-0068-7

[3] T. Netzsch and B. Jähne, "Ein Schnelles Verfahren zur Lösung des Stereokorrespondenz-Problems bei der 3DParticle Tracking Velocimetry," Proceedings 15. DAGM Symposium, Lübeck, September 1993, pp. 27-29.

[4] H.-G. Maas, "Complexity Analysis for the Determination of Image Correspondences in Dense Spatial Target Fields," International Archives of Photogrammetry and Remote Sensing, XXIX Part B5, 1992, pp. 102-107.

[5] G. A. Voth, A. La Porta, A. M. Crawford, J. Alexander and E. Bodenschatz, "Measurement of Particle Accelerations in Fully Developed Turbulence," Journal of Fluid Mechanics, Vol. 469, 2002, pp. 121-160. doi: $10.1017 / \mathrm{S} 0022112002001842$

[6] B. Lüthi, "Some Aspects of Strain, Vorticity and Material Element Dynamics as Measured with 3D Particle Tracking Velocimetry in a Turbulent Flow," Ph. D. Thesis, Swiss Federal Institute of Technology of Zürich, 2002.

[7] Y. Suzuki ans N. Kasagi, "Turbulent Air-Flow Measurement with the Aid of 3-D Particle Tracking Velocimetry in a Curved Square Bend," Flow, Turbulence and Combustion, Vol. 63, No. 1-4, 1999, pp. 415-442.

[8] H.-G. Maas, T. Putze and P. Westfeld, "Recent Developments in 3D-PTV and Tomo-PIV," In: W. Nitsche and C. Dobriloff, Eds., Imaging Measurement Methods for Flow Analysis, Springer, Heidelberg, 2009, pp. 53-62. doi:10.1007/978-3-642-01106-1_6

[9] A. V. Mikheev and V. M. Zubtsov, "Enhanced Particle Tracking Velocimetry (EPTV) with a Combined Two-Component Pair-Matching Algorithm," Measurement Science and Technology, Vol. 19, No. 8, 2008, pp. 1-16. doi:10.1088/0957-0233/19/8/085401

[10] H. S. Tapia, J. A. G. Aragon, D. M. Hernandez and B. B. Garcia, "Particle Tracking Velocimetry (PTV) Algorithm for Non-Uniform and Non-Spherical Particles," Electronics, Robotics and Automotive Mechanics Conference, Cuernavaca, September 2006, pp. 325-330. doi:10.1109/CERMA.2006.118

[11] L. Economikos, C. Shoemaker, K. Russ, R. S. Brodkey and D. Jones, "Toward Full-Field Measurements of Instantaneous Visualizations of Coherent Structures in Turbulent Shear Flows," Experimental Thermal and Fluid Science, Vol. 3, No. 1, 1990, pp. 74-86. doi:10.1016/0894-1777(90)90102-D

[12] Y. G. Guezennec, R. S. Brodkey, N. Trigui and J. C. Kent, "Algorithms for Fully Automated Three-Dimensional Particle Tracking Velocimetry," Experiments in Fluids, Vol. 17, No. 4, 1994, pp. 209-219. 


\section{doi:10.1007/BF00203039}

[13] C. Bendicks, D. Tarlet, B. Michaelis, D. Thévenin and B. Wunderlich, "Use of Coloured Tracers in Gas Flow Experiments for a Lagrangian Flow Analysis with Increased Tracer Density," 31st DAGM Symposium Proceedings, Heidelberg, 2009, pp. 392-401.

[14] R. Kuhn, R. Bordás, B. Wunderlich, B. Michaelis and D. Thévenin, "Colour Class Identification of Tracers Using Artificial Neural Networks," Proceedings of 10th International Conference on Engineering Applications of Neural Networks (EANN), Thessaloniki, 2007, pp. 387394.

[15] T. Etoh and K. Takehara, "Tracer Particles of Which Specific Gravity is Unity," In Proceedings Sixth International Symposium on Flow Visualisation, 1992, pp. 878881.

[16] R. Bordás, C. Bendicks, R. Kuhn, B. Wunderlich, D. Thévenin and B. Michaelis, "Coloured Tracer Particles Employed for 3D-PTV in Gas Flows," Proceedings of 13th International Symposium on Flow Visualization, Nice, France, 1-4 July 2008.

[17] R. Ramanath, W. E. Snyder, G. L. Bilbro and W. A. Sander, "Demosaicing Methods for Bayer Color Arrays," Journal of Electronic Imaging, Vol. 11, No. 3, 2002, pp. 306-315. doi:10.1117/1.1484495

[18] J. C. Crocker and D. G. Grier, "Methods of Digital Video Microscopy for Colloidal Studies," Journal of Colloid and
Interface Science, Vol. 179, No. 1, 1996, pp. 298-310. doi:10.1006/jcis. 1996.0217

[19] H.-G. Maas, "Digitale Photogrammetrie in der Dreidimensionalen Strömungsmesstechnik," Ph.D. Thesis, Swiss Federal Institute of Technology of Zürich, 1992.

[20] S. Nissen, "Neural Networks Made Simple" (Documentation of the FANN C/C++ Library 2005).

[21] J. Albertz and M. Wiggenhagen, "Guide for Photogrammetry and Remote Sensing," 5th Edition, Herbert-Wichmann Verlag, Heidelberg, 2009.

[22] T. Luhmann, S. Robson, S. Kyle and I. Harley, "Close Range Photogrammetry," Whittles Publishing, Dunbeath, 2006.

[23] V. G. S. Ernst, P. W. Sablik, J. Balendonck, Z. Houkes and P. P. L. Regtien, "A Surface Relief Meter Based on Trinocular Computer Vision," 5th International Conference on Image Processing and Its Applications, Edinburgh, 4-6 July 1995, pp. 281-285.

[24] T. Dracos, "Three-Dimensional Velocity and Vorticity Measuring and Image Analysis Techniques," Kluwer Academic Publishers, Dordrecht, 1996.

[25] D. Tarlet, C. Bendicks, Ch. Roloff, R. Bordás, B. Wunderlich, B. Michaelis and D. Thévenin, "Gas Flow Measurements by 3-D Particle Tracking Velocimetry Using Coloured Tracer Particles," Submitted in December 2010 to Flow, Turbulence and Combustion. 


\section{Appendix: Theoretical Prevision of Ambiguities}

A significant problem when using imaging measurement methods in 3-D flows is the occurrence of ambiguities during the spatial correspondence analysis. This is particularly true when a high number density of tracer particles is required i.e., when resolving small-scale flow structures. The correlation of particles between different images at a given time is mainly based on geometrical conditions such as the epipolar geometry. The intersecttion between the image plane and a plane formed by the object point and the perspective centers of the cameras should form a line. A corresponding tracer can only be found along this line. This decreases the search area from 2-D (the whole image) to 1-D (a line in the image). Using more than two cameras, the search space becomes smaller and smaller. Nevertheless, the ambiguities cannot be completely avoided in practice.

The same problem arises when correspondence analysis is performed in time to deduce information concerning the Lagrangian description of the flow field. Trajectories should be as long as possible without any interrupttion [2]. A restriction of the search space when considering successive time steps can be derived from physical considerations, taking only into account acceptable variations of velocity and acceleration and the local correlations between velocity vectors.

In what follows, the spatial correspondence problem is analyzed in greater detail. The formula to calculate the total number of expected ambiguities $N_{a}$ in a three-camera arrangement is given by (15) [4] and is also illustrated in Figure 10.

$$
N_{a}=\frac{4 \cdot\left(n^{2}-n\right) \cdot \varepsilon^{2}}{F \cdot \sin \alpha} \cdot\left(1+\frac{b_{12}}{b_{23}}+\frac{b_{12}}{b_{13}}\right)
$$

with

$n$ total number of tracers in image

$F \quad$ image size

$\alpha \quad$ intersecting angle between epipolar lines in the third image

$\varepsilon \quad$ tolerance of the epipolar band

$b_{x y} \quad$ distance between camera $x$ and camera $y$

The number of ambiguities becomes minimal when the cameras are arranged in an equilateral triangle so that $b_{12}=b_{13}=b_{23}$, and $\alpha=60^{\circ}$. Under this condition, used in the present experimental setup, the term in brackets is simply replaced by the factor 3 . Note that the number of ambiguities grows with the square of the number of tracers. An introduction of color classes for tracers acts like a reduction of the tracer particle number density. This reduces the number of ambiguities when the set of particles is separated by color into individual subsets. Assuming

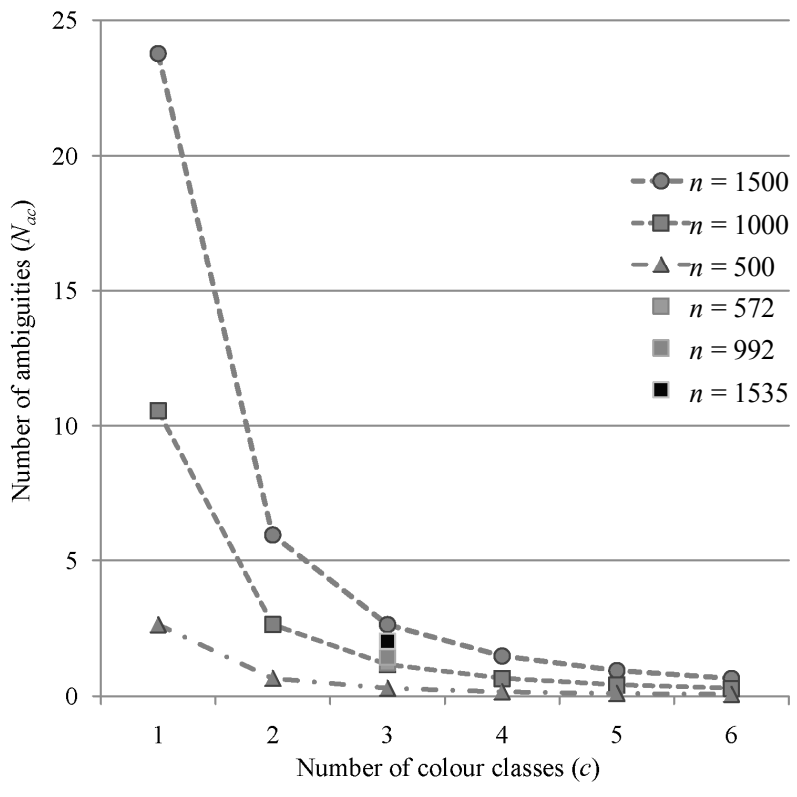

Figure 10. Theoretical reduction of the number of ambiguities when using up to six color classes (cf. Table 9). For comparison, the actual measured number of ambiguities for different numbers of particles $(n=572,952,1535)$ when using three color classes is also plotted (cf. Table 7).

Table 9. Number of ambiguities predicted by (16), see also Figure 11.

\begin{tabular}{ccccccc}
\hline$c$ & 1 & 2 & 3 & 4 & 5 & 6 \\
\hline$N_{a c}(c, n=500)$ & 2.64 & 0.66 & 0.29 & 0.16 & 0.10 & 0.07 \\
$N_{a c}(c, n=1000)$ & 10.56 & 2.64 & 1.17 & 0.66 & 0.42 & 0.29 \\
$N_{a c}(c, n=1500)$ & 23.77 & 5.94 & 2.64 & 1.48 & 0.95 & 0.66 \\
\hline
\end{tabular}

that the colored particles are uniformly distributed in camera images, the number of tracers $n_{c}$ of a particular color in each subset is $n / c$, where $c$ is the number of used colors. Since the correspondence problem is solved for each subset, the number of ambiguities $N_{a c}$ decreases as the square of the number of color classes (replacing $n$ by $n_{c}=n / c$ in $\left.(15)\right)$ :

$$
N_{a c}=\frac{12 \cdot\left(n_{c}^{2}-n_{c}\right) \cdot \varepsilon^{2}}{F \cdot \sin \alpha} .
$$

Results obtained from this equation are listed in Table $\mathbf{9}$ and plotted in Figure $\mathbf{1 0}$ using the parameters used in the present experiments: $F=1280 \times 1024$ pixels, $\alpha=60^{\circ}$ and $\varepsilon=1$ pixel considering up to six color classes $c$ and three different values for the number of tracers $n$ (500, 1000 and 1500). It can be seen in Figure 10 that the number of ambiguities is theoretically reduced by typically $80 \%$ when 3 color classes are taken into account. 